\title{
An Approach for Dynamic Characterisation of Passive Viscoelasticity and Estimation of Anthropometric Inertia Parameters of Paraplegic's Knee Joint
}

\author{
B.S. K. K. Ibrahim, M.S. Huq, M.O. Tokhi and S.C. Gharooni \\ Additional information is available at the end of the chapter
}

http://dx.doi.org/10.5772/45994

\section{Introduction}

Functional electrical stimulation (FES) is a promising way to restore mobility to individuals paralyzed due to spinal cord injury (SCI). Modelling and parameter identification of both the passive and active joint properties are needed to improve control of this nonlinear time varying system. In order to develop a suitable control strategy for the FES to move of the leg correctly, a proper model of stimulated muscle has to be used. The muscle is assumed to consist of two components: an active force generator and parallel passive properties. Riener and Edrich (1999) suggested passive muscle properties should be identified separately from active muscle properties because it is easier to consider the passive elastic forces as contributions to the total joint moment. Other researchers such as Zajac (1989) and Pandy et al. (1990) used a musculo-tendon model, in which the passive and the active forces are generated by single muscles. However, such models have too many parameters that cannot be identified non-invasively due to the muscle-joint redundancy of the musculoskeletal system (Riener and Edrich, 1999).

Conventionally the joint passive resistance is modelled as an elastic element like a torsion spring and a viscous element like a rotary damper (Lamb et al., 1991). These two resistances are non-linear, but the viscous resistance is often approached as a linear function of the joint angular velocity (Chizeck et. al., 1999; Mansour and Audu, 1986). These characteristics are important to estimate muscle load or fatigue during motion, especially in the field of biomechanics. Some researchers have shown further that these resistances influence the basis of motion effectively. For example, the resistance imposed by passive joint properties can impede the functionality of FES systems during limb movements (Amankwah, 2001). 
Segment mass of human body is an elementary inertial parameter for kinetic analyses of human motion. Many methods exist to estimate body segment properties. In the past, the most popular approach used to estimate segment parameters has been based on data obtained from elderly male cadavers. This database is quite limited in that a small number of cadavers have been studied. Thus, the database for making inertial estimates is not representative of the subjects often under investigation in many exercise and sport biomechanics studies. Dempster (1956) addressed the problem of mass model using cadaveric studies to establish segmental masses expressed as a percentage of total body mass (Winter, 1990). Other techniques have been developed in which inertial properties are directly measured for an individual. Zatsiorsky and Seluyanov (1985) used gamma mass scanning as a means of quantifying mass distribution in analyzing human body segment inertial characteristics. Both of these methods were promising and widely used but gave different measurement of the segment mass since calculated properties can vary drastically depending on the method used. Segment properties can significantly affect such variables, especially during swing phase (Doane and Quesada, 2006).

The pendulum test of Wartenberg is a technique commonly applied to evaluate passive properties in which the leg is allowed to drop from an initially extended position under the influence of gravity and then allowed to oscillate freely (Wartenberg, 1951). The test is very attractive in that it requires no special equipment and is very simple and the oscillatory movements of the lower leg recorded at the knee joint, are captured with electrogoniometers (Bajd and Vodovnik, 1984; Le Cavorzin et al., 2001), uniplanar video-based methods (Jamshidi and Smith, 1998) and 3D motion analysis systems (White et al., 2007). Most of the analyses of the pendulum motion depend on a second-order linear model to extract the elastic and viscous moments from the recorded leg oscillations. However a second order linear model does not provide an adequate description of the motion for either spastic or normal legs (Lin and Rymer, 1991).

In this paper a new approach for estimating passive properties of the paraplegic's knee joint based on pendulum test is described. On the basis of these experimental and optimization results, a non-linear fuzzy model is proposed which can be used to estimate the passive viscoelastic knee joint moment as a function of knee angle and knee velocity. The model of a dynamic system of the lower limb is derived using Kane's equations (Josephs and Huston, 2002) with accessibility to estimate of the foot mass, shank mass, moment of inertia about $\mathrm{COM}$ and position of COM along the segmental length of the subject.

\section{Material and methods}

The passive behaviour of the knee joint depends on the knee joint elastic moment $\left(M_{\mathrm{s}}\right)$ and the viscous moment $\left(M_{\mathrm{d}}\right)$. Considering the inertial $\left(M_{\mathrm{i}}\right)$ and gravitational $\left(M_{\mathrm{g}}\right)$ moments, a moment balance equation can be written as (Ferrarin and Pedotti, 2000):

$$
M_{\mathrm{i}}=M_{\mathrm{g}}+M_{\mathrm{s}}+M_{\mathrm{d}}
$$


or

$$
M_{\mathrm{i}}-M_{g}=M_{\mathrm{s}}+M_{\mathrm{d}}
$$

In this research the $M_{\mathrm{i}}-M g$ represented by equation of motion for dynamic model of the lower limb and $M_{\mathrm{s}}+M_{\mathrm{d}}$ represented by fuzzy model as viscoelasticity. The subject was a 48 year-old T2\&T3 incomplete paraplegic male with 20 years post-injury with height $=173 \mathrm{~cm}$ and weight $=80 \mathrm{~kg}$. Informed consent was obtained from the subject.

In this section, first the procedure to perform the pendulum test is presented to get the experimental data. Second, the equations of motion for dynamic model of the lower limb are introduced. Third, the estimations of anthropometric inertia parameters lower limb model are described briefly. Forth, the optimization of fuzzy model as passive viscoelasticity is outlined. Lastly new method for estimation and optimization of passive properties using GA by comparing with experimental data is elaborated. The procedure for estimation of the anthropometric inertia parameters and optimisation of FIS as passive viscoelasticity of the knee joint model are shown in Figure 1.

\subsection{Pendulum test}

Pendulum test can be used to evaluate passive properties such as viscosity and elasticity moments of the knee. Viscoelasticity is combination of elasticity and viscosity and represents passive resistances to joint motion associated with the structural properties of the joint tissue and of the muscular-tendon complex. Elasticity can be considered as an intrinsic property of the tissue to resist deformation, while viscosity is related to cohesive forces between adjacent layers of tissues. Both parameters may influence the joint range of motion affecting knee angle (Valle et. al, 2006). A genetic optimization algorithm is used to identify the unknown viscoelasticity by minimizing the error between the data obtained experimentally and from the simulation model. The pendulum test was conducted to measure the passive knee motion of an SCI patient. The subject sat on a chair, which allowed the lower leg to swing freely, while ankle joint was monitored to be at $0^{\circ}$. Reflexive or voluntary activation of muscles acting on the knee occurred during the pendulum test has been monitored to avoid the influence of pendulum movements.

In the pendulum test the knee was slowly extended, by having the experimenter lift it with minimal acceleration at the starting position (1) and then it was released as shown in Figure 3. The knee angle was recorded using electro-goniometer until the final position (2). A Biometric electro-goniometer was used to measure knee movements with sampling time of $0.05 \mathrm{~s}$. The electro-goniometer arrangement is shown in Figure 4.

\subsection{Equations of motion for dynamic model of the lower limb}

The inertial $\left(M_{\mathrm{i}}\right)$ and gravitational $\left(M_{\mathrm{g}}\right)$ moments are represented by mathematical model of a dynamic system of the lower limb based on Kane's equations as follows:- 


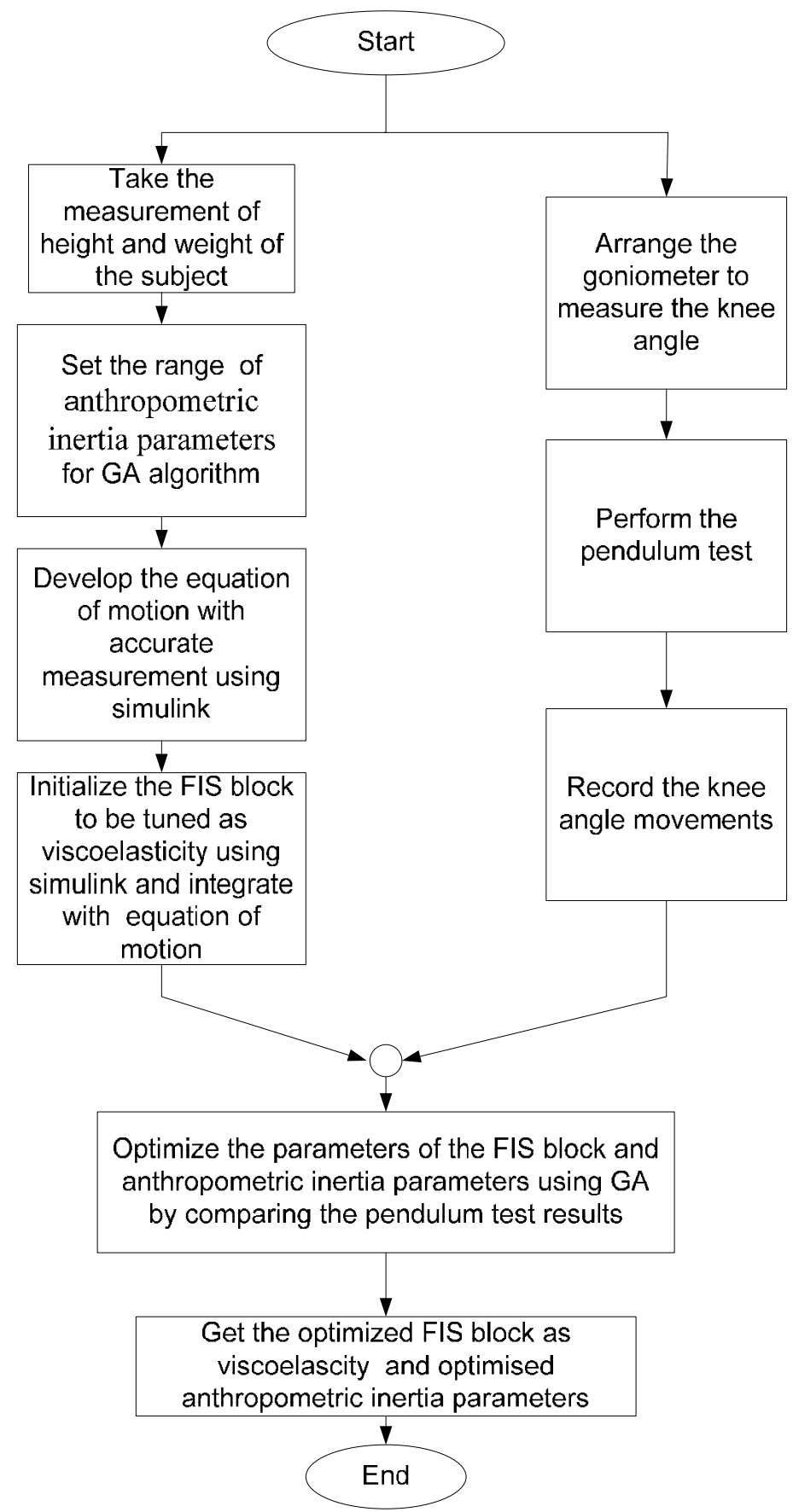

Figure 1. Optimization and Estimation Procedure 


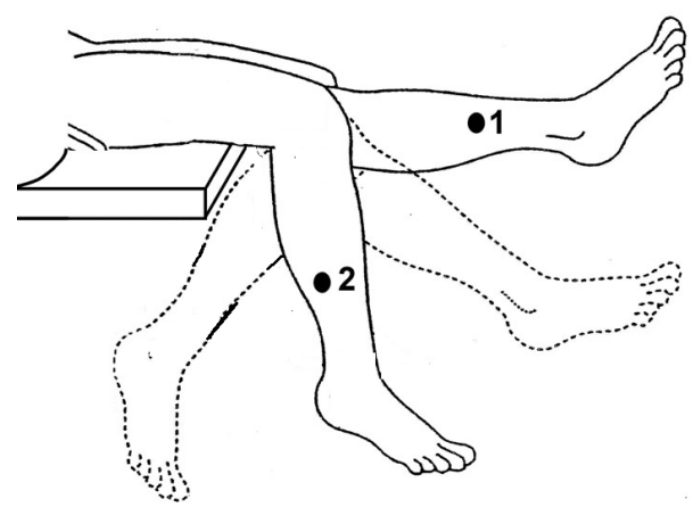

Figure 2. Limb oscillation during pendulum test (Valle et. al, 2008)

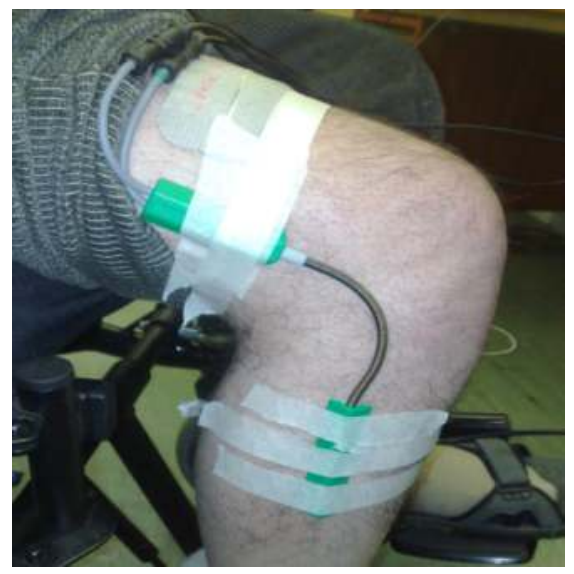

Figure 3. Electro-goniometer arrangement for pendulum test

$$
M_{i}-M_{g}=-m_{2} q_{2} \dot{\theta}_{1}^{2} r_{2}+m_{1} g \cos \theta_{1} r_{1}-I_{1} \ddot{\theta}_{1}+m_{2} g \cos \theta_{1} q_{2}-m_{1} r_{1}^{2} \ddot{\theta}_{1}-m_{2} q_{2}^{2} \ddot{\theta}_{1}
$$

where,

$m_{1}=$ shank mass, $m_{2}=$ foot mass, $I_{1}=$ moment of inertia about COM, $q_{2}=$ shank length $r_{1}=$ position of COM along the shank, $r_{2}=$ position of COM along the foot, $\theta_{1}=\mathrm{knee}$ angle, $\dot{\theta}_{1}=$ knee velocity, $\ddot{\theta}_{1}=$ knee acceleration, $\theta_{2}=$ ankle angle $g=$ gravity.

The lower limb model with the angles sign convention is shown in Figure 4. Anthropometric measurements of length of the lower limb were made as shown in Table 1. 


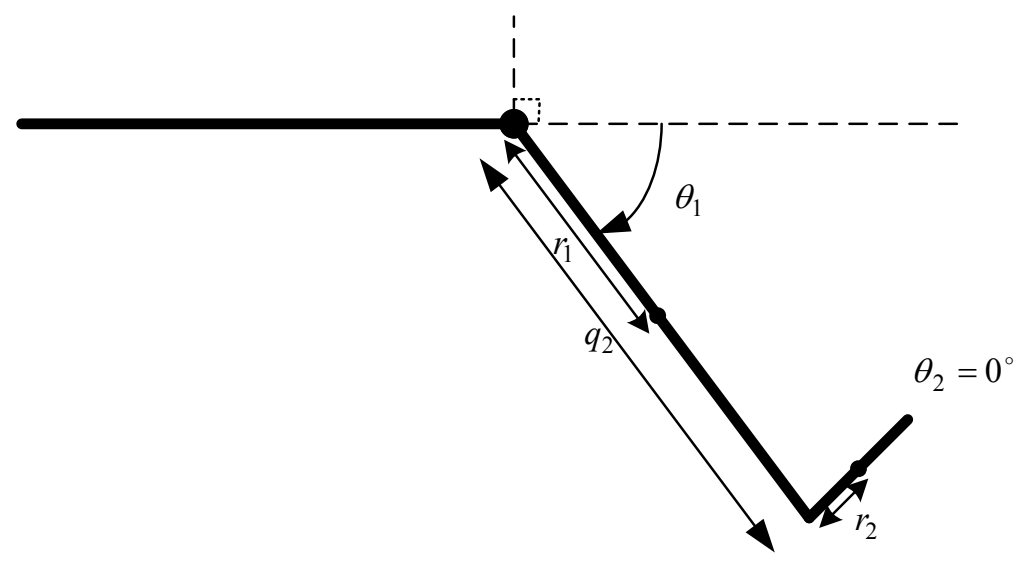

Figure 4. Lower limb model

\begin{tabular}{|l|l|}
\hline Segment & Length $(\mathrm{m})$ \\
\hline Shank & 0.4256 \\
\hline Foot & 0.0675 \\
\hline Approximated position of COM of shank & 0.2128 \\
\hline Approximated position of COM of soot & 0.03375 \\
\hline
\end{tabular}

Table 1. Anthropometric data of subject

\subsection{Estimation of anthropometric inertia parameters}

The lower limb dynamics is complex and less well defined because the form is not composed of simple geometrical shapes. Indeed, even the location of the mass center of elemental parts is imprecise, and comprehensive analyses of joint kinematics are extremely difficult (Josephs and Huston, 2002). To quantify limb dynamics, accurate estimates are needed of anthropometric inertia parameters (mass, COM location, and moments of inertia). Therefore these equations have accessed to optimise mass, moment of inertia about COM and position of COM along the segmental length of the subject using GA tuning method to fit the experimental data.

\subsubsection{Moments of Inertia}

Moments of inertia are fundamental parameters describing the mass distribution of body segments which enter into all computations involving segmental rotations. Methods based on the geometrical segment body models and appropriate anthropometric measures were used in measuring the moment of inertia of various segments in cadavers and in living subject (Dempster, 1955; Hatze, 1980; Jensen, 1986; Schneider and Zernicke, 1992). Different procedures were applied to identify the segment moment of inertia from the dynamic equation of motion. There is no unique way of calculating the moment of inertia To obtain 
more accurate moment of inertia of the knee, the minimum $\left(0.32 \mathrm{Nm}^{2}\right)$ and maximum $(0.58$ $\mathrm{Nm}^{2}$ ) range as reported by M. K. Lebiedowska (2003) is optimized using GA.

\subsubsection{Mass and position of COM of shank and foot}

Vatnsdal et al (2008) addressed the masses and positions of the COM of the lower limb are possible error source in the lower limb model. Dempster (1956) estimated the mass from the total height and weight of the person using standard human dimensions (Winter, 1990). Zatsiorski and Seluyanov (1983) estimated the mass on the basis of regression equations based on statistics obtained from measuring cadavers. However, both of the methods give different measurements for a selected subject as shown in Table 2. Therefore they can potentially introduce large errors, especially while modelling the passive properties, as the pendulum test results may be affected by those parameters. Thus, fine tuning these parameters around the predicted values with an efficient stochastic search algorithm such as GA could lead to good solution with immense potential of extracting accurate subject specific results. GA is used to optimise the foot and shank mass between the two measurements with $\pm 10 \%$ tolerance. While the approximated positions of COM along the segment length are assumed as in the centre of the segment length. To get the accurate positions, the position of the COM of shank and foot are optimised between $\pm 10 \%$ tolerances.

\begin{tabular}{|l|l|l|}
\hline Segment Mass & $\begin{array}{l}\text { Zatsiorski and } \\
\text { Seluyanov's Method }\end{array}$ & $\begin{array}{l}\text { Dempster's } \\
\text { Method }\end{array}$ \\
\hline Shank & $1.0499 \mathrm{~kg}$ & $1.16 \mathrm{~kg}$ \\
\hline Foot & $3.3973 \mathrm{~kg}$ & $3.72 \mathrm{~kg}$ \\
\hline
\end{tabular}

Table 2. Mass of patient using different methods

\subsection{Optimization of fuzzy model as passive viscoelasticity}

Modelling based on conventional mathematical tools is not well suited for dealing with complex and nonlinear nature systems. By contrast, a fuzzy inference system employing fuzzy 'if-then' rules can model the qualitative aspects of human knowledge and reasoning processes without employing precise quantitative analyses (Dinakaran, 2009). Besides that, the purpose of modeling passive viscoelascity is to be used as a part of the simulation platform and need to integrate with the active properties of the knee joint model for the controller application. Controller such as fuzzy control, neural network does not require the mathematical model of the plant. Therefore fuzzy model are well suited for modeling nonlinear models such as passive viscoelasticity. The passive viscoelastic joint moment is represented as a non-linear function of knee angle and knee angular velocity in a fuzzy model. An automatic Mamdani fuzzy system design method integrates three stages; determines membership functions, the rule-consequent parameters, and scaling factor at the same time. Gaussian membership functions are preferred, because of their continuously differentiable curves and smooth transitions. There are 58 parameters to optimise including 
30 parameters that determine the centre and width of the 15 Gaussian membership functions, 25 weights associated with the fuzzy rules, and 3 scaling factors for the normalization and denormalization of 2 inputs and 1 output of fuzzy model respectively. The weights of the 25 fuzzy rules have been optimised between 0 and 1 . The rules are identified based on expert knowledge that refers to relationship between knee angle and velocity and passive torque. Table 3 summarizes the rule base for the fuzzy model encompassing possible AND combinations of the input fuzzy values.

\begin{tabular}{|c|c|c|c|c|c|c|}
\hline & \multicolumn{5}{|c|}{ Knee angular velocity } \\
\hline & & NB & NS & $\mathrm{ZO}$ & PS & $\mathrm{PB}$ \\
\hline \multirow{5}{*}{ 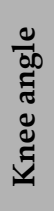 } & NB & PB & PB & PB & PS & $\mathrm{ZO}$ \\
\hline & NS & PB & PB & PS & $\mathrm{ZO}$ & NS \\
\hline & $\mathrm{ZO}$ & PB & PS & $\mathrm{ZO}$ & NS & NB \\
\hline & PS & PS & $\mathrm{ZO}$ & NS & NB & NB \\
\hline & PB & $\mathrm{ZO}$ & NS & NB & NB & NB \\
\hline
\end{tabular}

Table 3. Rule base of the fuzzy model $\mathrm{NB}=$ Negative big NS=Negative small $\mathrm{Z}=$ Zero PS=Positive small PB=Positive big

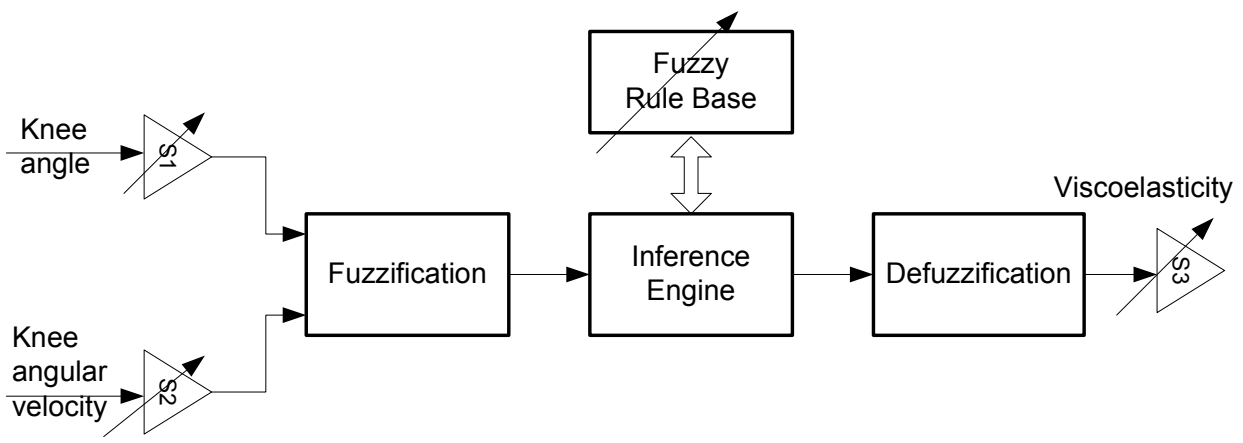

Figure 5. A fuzzy expert system model

The configuration of the fuzzy expert system model is shown in Figure 5. In the fuzzification step, crisp inputs are fuzzified into linguistic values to be associated to the input linguistic variables. After fuzzification, the inference engine refers to the fuzzy rule base containing fuzzy IF-THEN rules to derive the linguistic values for the intermediate and output linguistic variables. Once the output linguistic values are available, the defuzzifier produces the final crisp values from the output linguistic values. The defuzzification method was based on calculating the centre of gravity of the fuzzy output. Scaling factors is applied to ensure that the domain of discourse covers the whole range (Reznik, 1997). Therefore, two input scaling factors are used to transform the crisp inputs into the normalised inputs so as to keep their value within -1 and +1 . The scaling factors are S1 for knee angle, $\theta$ and S2 for knee angular velocity, $\dot{\theta}$. An output scaling factor S3 provides a transformation of the 
defuzzified crisp output from the normalised universe of the model output into an actual physical output (passive torque, $\tau_{p a s}$ ). These scaling factors influence the performance of the model and optimise simultaneously using GA. The ranges of the scaling factors are set as follows:-

a. S1 is between $1 / \theta_{\max }$ and $1 / \theta_{\min }$ of the experimental data.

b. S2 is between $1 / \dot{\theta}_{\max }$ and $1 / \dot{\theta}_{\min }$ of the experimental data.

c. $\quad$ S3 is between 1 and 50 .

\subsection{Estimation and optimization of passive properties using GA}

The GA is based on natural selection and population genetics theory (Goldberg, 1989). This evolutionary algorithm is chosen to estimate passive knee joint properties of paraplegic because the search space is large and complex. The advantage of the GA approach is robust, searches many points simultaneously, and is able to avoid local optima that the traditional algorithms might get stuck in (Lee, 1993).

GA is used to estimate a dynamic characterization of passive viscoelasticity of the knee joint using fuzzy model and estimation of the anthropometric inertia parameters such as foot mass, shank mass, moment of inertia about COM and positions of COM along the segmentals length of the lower limb as shown in Figure 6. The goal of GA optimization process is to minimize the error between the knee angle obtained experimentally and and from the model. The output prediction error is defined as:-

$$
e(t)=y(t)-\hat{y}(t)
$$

where $y(t)$ is the experimental data and $\hat{y}(t)$ is the estimated current output of knee angle. The 'goodness of fit' of the identified model is determined using the objective function by minimizing the mean-squared error (MSE);

$$
f=\min \left\{\frac{\sum_{i=1}^{N}(y(t)-\hat{y}(t))^{2}}{N}\right\}
$$

The GA optimization procedure is shown in Figure 7 and implemented in MATLAB with GA Toolbox. First, an initial population of individuals is generated. Each individual corresponds to a chromosome, which is a set of specific genes from the biological point of view. Then, the performance of each member of the population is assessed through an objective function imposed by the problem. This fires the process of selecting pairs of individuals which will be mated together during reproduction. Selection is based probabilistically on a gene's fitness value; the higher the fitness of a gene, the more likely it can reproduce. After selecting two parents, crossover is performed according to a crossover probability. If crossover is to be performed, offspring are constructed by copying portions of 


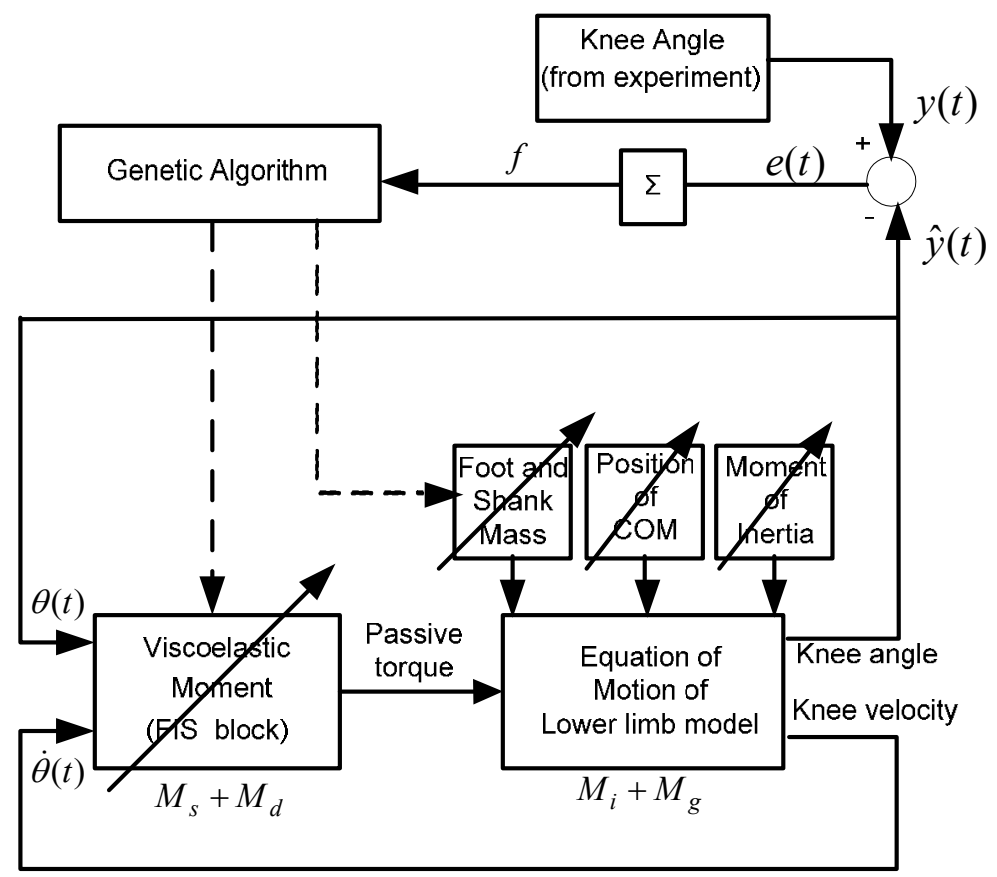

Figure 6. Optimization of passive properties

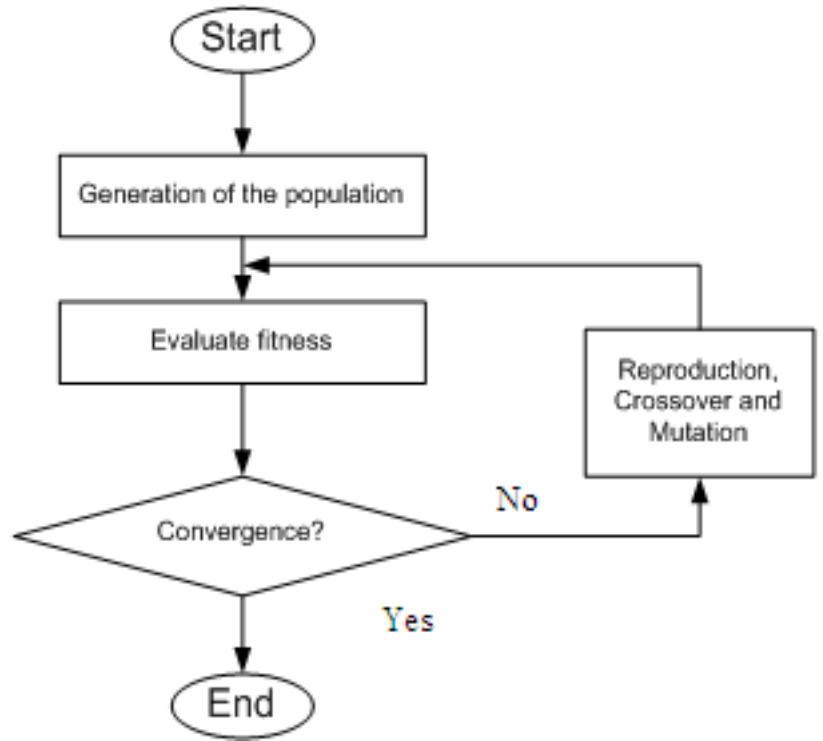

Figure 7. The GA optimization procedure 
parent genes designated by random crossover points. Otherwise, an offspring copies its entire gene from one of the parents. As each bit is copied from parent to offspring, the bit has the probability of mutating. Mutation is believed to help in reinjection of any information that may have been lost in previous generations (Goldberg, 1989).

\section{Results}

In this section, the results of the new method to model the passive viscoleasticity and estimate the anthropometric inertia parameters by GA optimization process are presented. Population size of GA was set to 50 and crossover and mutation probabilities were 0.8 and 0.001 respectively. The automatic GA optimization process was generated up to 200 generations of solutions. The best solution was kept and the rest were discarded until there is no significant change in the mean square error (MSE) was observed after the $165^{\text {th }}$ generation. The minimum MSE achieved was 1.87 . The response of the model was tested and the result is shown in Figure 8. The results showed that the model parameters were estimated well and that the fit between the model and the experimentally data was good.

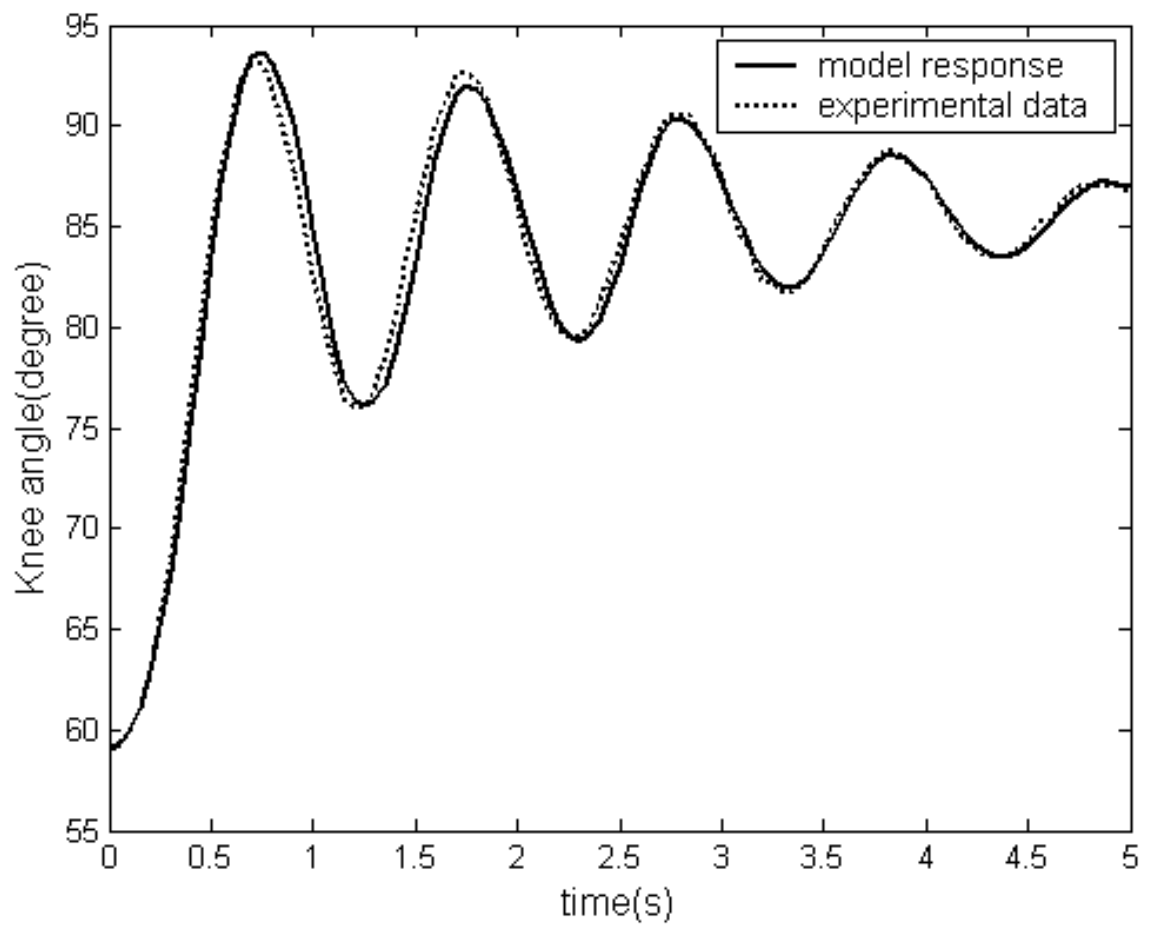

Figure 8. Responses of the pendulum test and the model response 


\subsection{Optimised value of anthropometric inertia parameters}

The optimized value of the anthropometric inertia parameters such masses, moment of inertia about COM and positions of COM along the segmentals length of the subject's lower limb are tabulated in Table 4 . The accuracy of these optimised anthropometric inertia parameters been validated through repeated process of optimisation.

\begin{tabular}{|l|l|}
\hline Parameter & Optimised Value \\
\hline Foot Mass & $0.95 \mathrm{~kg}$ \\
\hline Shank Mass & $3.5 \mathrm{~kg}$ \\
\hline Moment of Inertia & $0.35823 \mathrm{Nm}^{2}$ \\
\hline Position of COM of Foot & $0.035 \mathrm{~m}$ \\
\hline Position of COM of Shank & $0.22 \mathrm{~m}$ \\
\hline
\end{tabular}

Table 4. Optimised Value ofAnthropometric Inertia Parameters

\subsection{Optimised Fuzzy Model as Viscoelasticity}

The vicoelasticity is represented by a fuzzy model. The GA optimization is used to optimise 48 parameters including 30 associated with the membership function, 15 weights associated with the minimized fuzzy rules and 3 scaling factors of fuzzy model. The scaling factor for the normalization and denormalization of two inputs and output were $0.0091247,0.0053982$ and 35 respectively. The fuzzy model takes into account the nonlinear component of passive viscoelasticity. A three-dimensional plot that represents the mapping from knee angle and knee angular velocity to viscoelastic moment is shown in Figure 9. This surface plot shows the normalised viscoelasticity (unit less) changes as a function of the normalised knee angle (unit less) and normalised velocity (unit less). The presence of the non-linearities in the viscoelasticity can be noted on this uneven surface shape from both angles. The valley for knee angle between 0.5 and 1 gives high viscoelasticity could be due to the high stiffness near extended knee joint.

\subsection{Model validation}

Model validation is possibly the most important step in the modelling process. The model and the optimised parameters obtained from the optimization process were validated in terms of consistency and the prediction error. Two different approaches of model validity are conducted. Different sets of experimental conditions are usually required to define the domain of a model's intended applicability. Therefore, in the first validation process, the model is validated with a different set of data than the training data. To avoid any change in the actual plant, the validation data is obtained in the same experimental arrangement but with a different initial knee joint angle. The responses of the optimised model and new experimental data are shown in Figure 10. It is noted that the two agree closely with one another. 


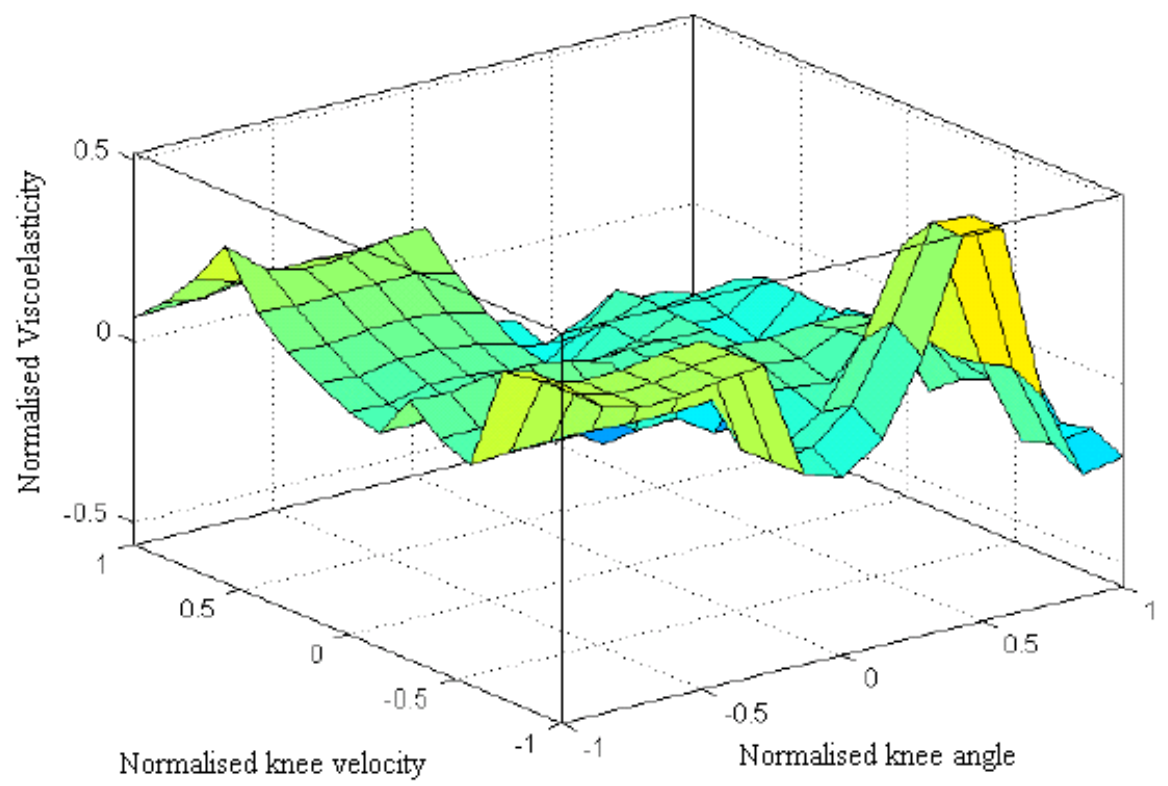

Figure 9. 3D Fuzzy surface of normalised viscoelastic moment

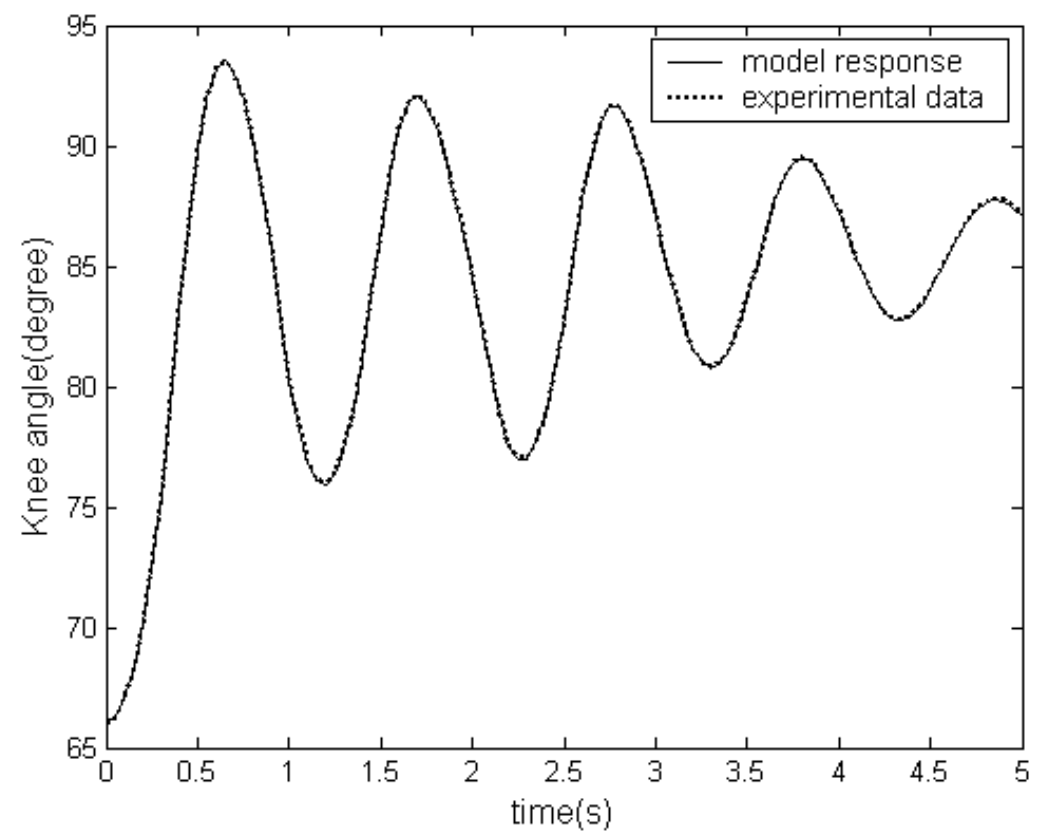

Figure 10. Result of first validation 
Second, the optimised segmental masses are validated by repeating the same optimization process for further four times. The five sets of the optimised parameters emanating from five different runs of the GA routines are shown in Table 5. The results of the optimised anthropometric inertia parameters from different simulation runs exhibit acceptable repeatability with only a slight difference between each other with small standard deviation. Therefore, it can be concluded that the optimised masses obtained are valid.

\begin{tabular}{|l|c|c|c|c|c|c|}
\hline Iteration & $\begin{array}{c}\text { Foot } \\
\text { Mass } \\
\mathbf{( k g )}\end{array}$ & $\begin{array}{c}\text { Shank } \\
\text { Mass } \\
\mathbf{( k g}\end{array}$ & $\begin{array}{c}\text { Moment of } \\
\text { inertia }\left(\mathrm{Nm}^{2}\right)\end{array}$ & $\begin{array}{c}\text { Position of } \\
\text { COM of } \\
\text { Foot } \mathbf{( m )}\end{array}$ & $\begin{array}{c}\text { Position of } \\
\text { COM of } \\
\text { Shank (m) }\end{array}$ \\
\hline 1 & 0.95 & 3.5 & 0.35823 & 0.0350 & 0.220 \\
\hline 2 & 0.948 & 3.51 & 0.35830 & 0.0352 & 0.221 \\
\hline 3 & 0.951 & 3.49 & 0.35828 & 0.0355 & 0.220 \\
\hline 4 & 0.949 & 3.51 & 0.35815 & 0.0351 & 0.222 \\
\hline & 5 & 0.952 & 3.49 & 0.35820 & 0.0345 & 0.221 \\
\hline Mean & $\mathbf{0 . 9 5}$ & $\mathbf{3 . 5}$ & $\mathbf{0 . 3 5 8 2}$ & $\mathbf{0 . 0 3 5 1}$ & $\mathbf{0 . 2 2 0 8}$ \\
\hline $\begin{array}{l}\text { Standard } \\
\text { deviation }\end{array}$ & $\mathbf{0 . 0 0 1 6}$ & $\mathbf{0 . 0 1}$ & $\mathbf{0 . 0 0 0 0 6}$ & $\mathbf{0 . 0 0 0 3 6}$ & $\mathbf{0 . 0 0 0 8 3}$ \\
\hline
\end{tabular}

Table 5. Five sets of the optimised parameters

\section{Discussion}

In this approach the passive properties have been divided into two parts; First, mathematical model is used to represent the combination of knee joint inertial $\left(M_{\mathrm{i}}\right)$ and gravitational $\left(M_{\mathrm{g}}\right)$ moments. Second, fuzzy model is applied to represent the combination of the elastic moment $\left(M_{\mathrm{s}}\right)$ and the viscous moment $\left(M_{\mathrm{d}}\right)$ as viscoelastic moment of the knee joint. Mathematical model has been used because of the availability of this model and accessibility to estimate anthropometric inertia parameters. This could be an easy method to estimate these parameters without go through complicated clinical experiment since these parameters vary for each subject. The system such as viscoelasticity could be difficult to model due to complexity and nonlinerity. Therefore, fuzzy model is used to eliminate the development of complex mathematical model and helps to simplify the modelling task. These passive properties model then need to be integrated with active properties of the knee joint model to have a complete model of the knee joint. Finally, these models can be utilized as platform for the simulation purpose of the control system development.

\section{Conclusion}

A new approach of estimation of the anthropometric inertia parameters and model of the passive viscoelasticity of the knee joint has been presented. Fuzzy model has been used to model the passive viscoelasticity and could be an effective tool for the modeling of uncertain nonlinear systems without represents using complicated mathematical model. The 
anthropometric inertia parameters of the lower limb such as foot mass, shank mass, moment of inertia about COM and positions of COM along the segmental length of the lower limb have been optimized between the given ranges to obtain the accurate value of the equations of motion. These optimizations have been performed simultaneously using GA with the objective to minimize the error between prediction model and the experimental data. Each person has a unique composition of muscle tissue in their body therefore this passive properties are not generally applicable.

\section{Author details}

B.S. K. K. Ibrahim, M.S. Huq, M.O. Tokhi and S.C. Gharooni

Department of Automatic Control and System Engineering, University of Sheffield, United Kingdom

\section{References}

B. Vatnsdal, P. E. Ingvarson, D. Rafolt, V.Gudmudsdottir, S.Knutsdottir,S. Yngvason and T.Helgason, Monitoring of knee joint mechanics during electrical treatment of denervated degenerated muscle, Proceeding of the 13th annual conference of the IFESS, 2008.

C. Fonseca, and P. Fleming, "Genetic algorithms for multiobjective optimization: formulation, discussion and generalization," Genetic Algoritms: Proceeding of the Fifth International Conference, San Mateo, CA, pp. 416-423, 1993.

D.A.Winter. Biomechanics and motor control of human movement. 2nd edition, WileyInterscience, New York, 1990.

D.C. Lin, W.Z. Rymer. A quantitative analysis of pendular motion of the lower leg in spastic human subjects. IEEE Trans Biomed Eng. 1991 Sep;38(9):906-18.

D. E. Goldberg, Genetic algorithms in search, optimization and machine learning. Reading, MA: Addison-Wesley, 1989.

F.E. Zajac, Muscle and tendon properties: models, scaling, and application to biomechanics and motor control. CRC Critical Reviews in Biomedical Engineering 17, pp. 359-411, 1989.

H. Hatze, "A three-dimensional multivariate model of passive human torques adn articular boundaries," Clinical Biomechanics, vol. 12, pp. 128-135, 1997.

H.J. Chizeck, S. Chang, R.B. Stein, A. Scheiner, D.C. Ferencz, Identification of electrically stimulated quadriceps muscles in paraplegic subjects, IEEE Transactions Biomedical Engineering on Vol 46, Issue 1,pp:51 - 61, 1999.

H. M. Franken, P. H. Veltink, R. Tijsmans, H. Nijmeijer, and H. B. K.Boom, "Identification of passive knee joint and shank dynamics in paraplegics using quadriceps stimulation," Transactions on Rehabilitation Engineering, vol. 1,pp. 154-164, 1993.

H. White, T.L. Uhl, S. Augsburger, C.Tylkowski. Reliability of the three-dimensional pendulum test for able-bodied children and children diagnosed with cerebral palsy. Gait Posture 2007;26:97-105.

J. E. Doane and P. M. Quesada, Subject specific body segment parameter estimation using 2D work and energy principles, Gait \& Posture, Volume 24, Supplement 2, pp.123-125,2006. 
J. M. Mansour and M. L. Audu, The passive elastic moment at the knee and its influence on human gait, Journal Biomechical., vol. 19, no. 5, pp. 369-373, 1986.

J., F. Lamb, C. G.Ingram, I. A. Johnston, R. M. Pitman, Essentials of Physiology, Blackwell Scientific Publications, UK, 1991.

K. Amankwah, R.J. Triolo, R.F. Kirsch. The effects of spinal cord injury on lower limb passive joint moments revealed through a nonlinear viscoelastic model. Journal of Rehabilitation Research and Development 41 (1), 15-32, 2004.

L. Reznik, Fuzzy Controllers, Newnes-Butterworth-Heinemann, Oxford, 1997.

M. Ferrarin, and A. Pedotti, The relationship between electrical stimulus and joint torque: a dynamic model. IEEE Transactions on Rehabilitation Engineering, 8 (3), pp 342-352, 2000.

M. Jamshidi, A.W. Smith. Clinical measurement of spasticity using the pendulum test: comparison of electrogoniometric and videotape analyses. Arch Phys Medical Rehabilitation 1998;77:1129-32.

M. S. Valle, A. Casabona, R. Sgarlata, R. Garozzo, The pendulum test as a tool to evaluate passive knee stiffness and viscosity of patients with rheumatoid arthritis. BMC Musculoskeletal Disorder, 2006.

M.G. Pandy, F.E. Zajac, E.Sim, W.S. Levine, An optimal control model for maximum-height human jumping, Journal of Biomechanics 23, pp.1185-1198, 1990.

P.H. Veltink et al. Modeling the optimal control of cyclical leg movements induced by functional electrical stimulation, Int. J. Art. Org., vol. 15, pp. 746-755, 1992.

P. L. Cavorzin, X Hernot, O Bartier, G Carrault, P Rochcongar, F Chagneau. A computed model of the pendulum test of the leg for routine assessment of spasticity in man. ITBM-RBM 2001;22:170-7.

R. Massoud. Intelligent control techniques for spring assisted FES-cycling. PhD Thesis. The University of Sheffield, Sheffield, UK, 2007.

R. Riener and T. Edrich, "Significance of passive elastic joint moments in FES," Proceedings of the 2nd Conference of IFESS, pp. 103-106, 1997.

T. Bajd, L.Vodovnik, Pendulum testing of spasticity. Journal of Biomedical Engineering 1984;6:9-16.

V. Zatsiorsky and V. Seluyanov, "The mass and inertia characteristics of the human body", Biomechanics VIII-B, University Park Press, pp. 1152-1159, 1983.

W. T. Dempster. Space requirements of the seated operator. WADC Technical Report (TR-59159), Wright-Patterson Air Force Base, OH, 1955.

Wartenberg, R. (1951). Pendulousness of the leg as a diagnostic test. Neurology, 1, 18-24. 